\title{
Caracterização Matemática e Visualização da Esfera de Bloch: Ferramentas para Computação Quântica
}

L.M. CARVALHO ${ }^{1}$, Departamento de Matemática Aplicada, Instituto de Matemática e Estatística, UERJ, Rua São Francisco de Xavier, 524, sala 6026, bloco D, Maracanã, 20550-013 Rio de Janeiro, RJ, Brasil

C. LAVOR ${ }^{2}$, Departamento de Matemática Aplicada, IMECC, UNICAMP, CP 6065, 13081-970 Campinas, SP, Brasil

V.S. MOTTA 3 , Departamento de Ciências Fundamentais, Radiações e Meio Ambiente, Instituto Militar de Engenharia, Praça General Tibúrcio, 80, Praia Vermelha, 22290-270 Rio de Janeiro, RJ, Brasil.

Resumo. Faremos uma descrição matemática da esfera de Bloch, discutiremos propriedades dessa representação e apresentaremos algumas visualizações de 1 qbit.

\section{Introdução}

A visualização de propriedades matemáticas de um objeto é sempre bem-vinda. São inúmeros os exemplos em matemática e física: os eixos cartesianos, o plano de Argand-Gauss, os diagramas de Feynman, entre outros. A esfera de Bloch [4] é uma das representações possíveis, em três dimensões, para um bit quântico. No entanto, vários livros da área de computação quântica dão um tratamento pouco aprofundado à sua construção e às suas propriedades (por exemplo, ([13], p. 15) e ([15], p. 54)), e alguns outros nem mesmo o fazem ([6], [9]).

Nosso objetivo é produzir ferramentas que ajudem aos desenvolvedores de algoritmos quânticos, aos pesquisadores da área e aos estudantes interessados a ter maior facilidade de incorporar a visualização às propriedades algébricas e geométricas de estados quânticos emaranhados e não-emaranhados. Nesse sentido, um primeiro passo é a descrição matemática da esfera de Bloch e a representação visual de algumas propriedades relevantes.

Em computação quântica, ainda não há uma padronização de representações para um ou mais q-bits, mas dependendo do foco de cada trabalho, algumas representações vêm sendo desenvolvidas. Em [3], propõe-se uma representação para

\footnotetext{
${ }^{1}$ luizmc@gmail.com

${ }^{2}$ clavor@ime.unicamp.br

3 valeria@ime.eb.br
} 
$m$ q-bits usando-se uma generalização da esfera de Bloch, através da Álgebra de Clifford. A principal motivação desse trabalho é descobrir, se dada uma matriz Hermitiana com traço unitário, ela representa ou não um estado quântico. Em [5], usa-se Geometria Algébrica em um espaço real de seis dimensões para se construir grupos unitários especiais para dois q-bits, similar à representação de um q-bit pela esfera de Bloch. Em [12], usa-se o fibrado de Hopf para representar dois e três q-bits, buscando uma generalização para a esfera de Bloch no caso de dois q-bits. Em [11], a ação de um tipo de transformação unitária particular é representada na esfera de Bloch. Em [10], são apresentadas condições para se descrever medidas fisicamente realizáveis na esfera de Bloch. Citamos alguns outros trabalhos que buscam discutir representações, por exemplo, [1], [2], [7], [8], [16].

Nosso ponto de partida é simples e diferente dos referidos acima. Uma vez que todo circuito quântico pode ser decomposto em uma série de portas lógicas quânticas universais de apenas um ou dois q-bits, conseguir uma boa representação visual para esses fenômenos é uma contribuição para a área de algoritmos quânticos. Nesse artigo, estamos discutindo a representação e propriedades visuais para um q-bit, apresentando, inclusive, um exemplo não trivial de ortogonalidade entre vetores, sem existência de perpendicularismo. Em trabalhos futuros, pretendemos desenvolver representações para dois q-bits.

O restante desse artigo consta de uma descrição matemática detalhada da esfera de Bloch, onde demonstramos algumas de suas propriedades (seções 3. e 4.). Na seção 2., introduzimos definições preliminares (maiores detalhes em [14]). Para uma visão mais abrangente sobre computação quântica, sugerimos o livro de Nielsen e Chuang [13].

\section{Modelos para um q-bit}

Em computação clássica, a menor porção de informação disponível é o bit, que pode assumir apenas dois valores, por exemplo, 0 e 1. Em computação quântica, utilizam-se estados quânticos no lugar de estados clássicos. O bit é substituído pelo bit quântico, o $q$-bit, e os valores 0 e 1 de um bit são substituídos pelos vetores $|0\rangle$ e $|1\rangle$, representados por

$$
|0\rangle=\left[\begin{array}{l}
1 \\
0
\end{array}\right] \quad \text { e } \quad|1\rangle=\left[\begin{array}{l}
0 \\
1
\end{array}\right] .
$$

Essa notação, utilizada em mecânica quântica, é conhecida por notação de Dirac.

A principal diferença entre um bit e um q-bit é que um q-bit genérico $|\psi\rangle$ é uma combinação linear dos vetores $|0\rangle$ e $|1\rangle$, ou seja, $|\psi\rangle=\alpha|0\rangle+\beta|1\rangle$, onde $\alpha$ e $\beta$ são números complexos. Note que os vetores $|0\rangle$ e $|1\rangle$ pertencem ao espaço vetorial complexo $\mathbb{C}^{2}(\mathbb{C})$ e formam uma base ortonormal para esse espaço vetorial. Em computação quântica, essa base é chamada de base computacional e o vetor $|\psi\rangle$ é denominado de superposição dos vetores $|0\rangle$ e $|1\rangle$, com amplitudes $\alpha$ e $\beta$. Em mecânica quântica, vetor é também chamado de estado; usaremos os dois termos indistintamente.

A interpretação física de um q-bit é que ele está simultaneamente nos estados $|0\rangle$ e $|1\rangle$. Isso faz com que a quantidade de informação armazenada no estado 
$|\psi\rangle$ possa ser infinita. Entretanto, essa quantidade infinita de informação está no nível quântico. Para torná-la acessível, no nível clássico, precisamos fazer uma medida. A mecânica quântica diz que o processo de medida altera o estado de um q-bit, fazendo-o assumir o estado $|0\rangle$, com probabilidade $|\alpha|^{2}$, ou o estado $|1\rangle$, com probabilidade $|\beta|^{2}$. Com apenas duas possibilidades, $|0\rangle$ ou $|1\rangle$, temos então que $|\alpha|^{2}+|\beta|^{2}=1$. Calculando a norma de $|\psi\rangle$, obtemos: $\||\psi\rangle \|=\sqrt{|\alpha|^{2}+|\beta|^{2}}=1$. Como conseqüência imediata, podemos estabelecer o primeiro modelo para um qbit.

Proposição 2.1. Um vetor unitário de $\mathbb{C}^{2}(\mathbb{C})$ é um modelo matemático para um $q$-bit.

Um outro modelo é dado na proposição abaixo.

Proposição 2.2. Um vetor unitário de $\mathbb{R}^{4}(\mathbb{R})$ é um modelo matemático para um $q$-bit $|\psi\rangle=\alpha|0\rangle+\beta|1\rangle$ e a esfera unitária $\mathrm{S}^{3}$ de $\mathbb{R}^{4}$ é o lugar geométrico dos q-bits.

Demonstração. Considere $\alpha=a+\mathrm{i} b$ e $\beta=c+\mathrm{i} d(a, b, c, d \in \mathbb{R})$. Como $|\alpha|^{2}=$ $\left(\sqrt{a^{2}+b^{2}}\right)^{2}$ e $|\beta|^{2}=\left(\sqrt{c^{2}+d^{2}}\right)^{2}$, temos $a^{2}+b^{2}+c^{2}+d^{2}=1$. Nesse caso, interpretamos um q-bit como sendo um vetor unitário $(a, b, c, d)$ de $\mathbb{R}^{4}$. Como essa propriedade é válida para todos os q-bits, a esfera $\mathrm{S}^{3}$ é o lugar geométrico buscado.

Para introduzirmos a esfera de Bloch, precisamos do seguinte resultado.

Proposição 2.3. Um q-bit pode ser escrito na forma polar

$$
|\psi\rangle=\mathrm{e}^{i \gamma}\left[\cos (\xi)|0\rangle+\mathrm{e}^{i \varphi} \operatorname{sen}(\xi)|1\rangle\right]
$$

onde $0 \leq \gamma<2 \pi, 0 \leq \varphi<2 \pi$ e $0 \leq \xi \leq \pi / 2$.

Demonstração. Expressemos as amplitudes do q-bit $|\psi\rangle=\alpha|0\rangle+\beta|1\rangle$ em coordenadas polares. Temos $\alpha=|\alpha| \mathrm{e}^{i \operatorname{Arg}(\alpha)} \quad$ e $\quad \beta=|\beta| \mathrm{e}^{i \operatorname{Arg}(\beta)}, \operatorname{onde} \operatorname{Arg}(z)$ é o ramo principal do argumento do número complexo $z$, ou seja, $0 \leq \operatorname{Arg}(z)<2 \pi$ e $|z|$ é o seu módulo. Nesse caso, existe uma bijeção entre cada número complexo e essa representação polar.

Definindo $\gamma=\operatorname{Arg}(\alpha)$ e $\varphi=\operatorname{Arg}(\beta)-\operatorname{Arg}(\alpha)$, podemos reescrever o q-bit $|\psi\rangle=\alpha|0\rangle+\beta|1\rangle$ como (nesse artigo, todas as operações com ângulos devem ser consideradas em aritmética módulo $2 \pi)|\psi\rangle=|\alpha| \mathrm{e}^{i \gamma}|0\rangle+|\beta| \mathrm{e}^{i(\gamma+\varphi)}|1\rangle$. Sendo $|\alpha| \geq 0,|\beta| \geq 0$ e $|\alpha|^{2}+|\beta|^{2}=1$, podemos também definir $\xi$ por meio das equações $\cos (\xi)=|\alpha|$ e $\operatorname{sen}(\xi)=|\beta|$. Note que $0 \leq \xi \leq \pi / 2$. Finalmente, podemos escrever

$$
|\psi\rangle=\mathrm{e}^{i \gamma}\left[\cos (\xi)|0\rangle+\mathrm{e}^{i \varphi} \operatorname{sen}(\xi)|1\rangle\right]
$$

onde

$$
\begin{aligned}
& \xi=\arccos (|\alpha|)=\operatorname{arcsen}(|\beta|) \quad(0 \leq \xi \leq \pi / 2) \\
& \varphi=\operatorname{Arg}(\beta)-\operatorname{Arg}(\alpha) \quad(0 \leq \varphi<2 \pi) \\
& \gamma=\operatorname{Arg}(\alpha) \quad(0 \leq \gamma<2 \pi)
\end{aligned}
$$




\section{A Esfera de Bloch}

Em computação quântica, o escalar e $\mathrm{e}^{i \gamma}$, em (2.1), é denominado fator de fase global (veja [13], p. 93). Note que dois q-bits quaisquer $|\psi\rangle=\alpha|0\rangle+\beta|1\rangle$ e $\mathrm{e}^{\mathrm{i} \zeta}|\psi\rangle$ têm as mesmas probabilidades de, ao serem medidos, produzirem os estados $|0\rangle$ e $|1\rangle$, pois

$$
\mathrm{e}^{i \zeta}|\psi\rangle=\mathrm{e}^{\mathrm{i} \zeta} \alpha|0\rangle+\mathrm{e}^{\mathrm{i} \zeta} \beta|1\rangle \quad \text { e } \quad\left|\mathrm{e}^{\mathrm{i} \zeta} \alpha\right|=\left|\mathrm{e}^{\mathrm{i} \zeta}\right||\alpha|=|\alpha| .
$$

Note que o mesmo ocorre para $\beta$.

Sabemos, também, que a evolução de um q-bit é descrita, matematicamente, pela aplicação sucessiva de operadores unitários (veja [13], p. 81). Ao aplicarmos uma matriz unitária $U$ em um q-bit $|\psi\rangle=\mathrm{e}^{i \gamma}\left[\cos (\xi)|0\rangle+\mathrm{e}^{i \varphi} \operatorname{sen}(\xi)|1\rangle\right]$, obtemos

$$
U|\psi\rangle=\mathrm{e}^{i \gamma} U\left[\cos (\xi)|0\rangle+\mathrm{e}^{i \varphi} \operatorname{sen}(\xi)|1\rangle\right] .
$$

Note que o fator de fase global não se modifica pela aplicação de um operador unitário. Usando essas propriedades, podemos desprezar o fator $\mathrm{e}^{i \gamma} \mathrm{e}$, a partir daí, tentar encontrar uma representação geométrica para um q-bit em $\mathbb{R}^{3}$.

Proposição 3.1. Os vetores da forma $|\psi\rangle_{R}=\cos (\xi)|0\rangle+\mathrm{e}^{i \varphi} \operatorname{sen}(\xi)|1\rangle$, com $0 \leq$ $\xi \leq \pi / 2$ e $0 \leq \varphi<2 \pi$, pertencem a um subespaço vetorial de $\mathbb{C}^{2}(\mathbb{R})$ de dimensão três.

Demonstração. Reescrevendo o vetor $|\psi\rangle_{R}$, temos

$$
|\psi\rangle_{R}=\cos (\xi)|0\rangle+\mathrm{e}^{i \varphi} \operatorname{sen}(\xi)|1\rangle=\cos (\xi)|0\rangle+\cos (\varphi) \operatorname{sen}(\xi)|1\rangle+\mathrm{i} \operatorname{sen}(\varphi) \operatorname{sen}(\xi)|1\rangle .
$$

Ou seja, o vetor $|\psi\rangle_{R}$ pode ser visto como

$$
|\psi\rangle_{R}=\left[\begin{array}{c}
a \\
c+\mathrm{i} d
\end{array}\right]
$$

$\operatorname{com} a, b, c \in \mathbb{R}$.

O espaço vetorial real $\mathbb{C}^{2}(\mathbb{R})$ tem dimensão quatro e uma de suas bases ortonormais é o conjunto

$$
\left\{\left[\begin{array}{l}
1 \\
0
\end{array}\right],\left[\begin{array}{l}
\mathrm{i} \\
0
\end{array}\right],\left[\begin{array}{l}
0 \\
1
\end{array}\right],\left[\begin{array}{l}
0 \\
\mathrm{i}
\end{array}\right]\right\}
$$

Entretanto, podemos representar o vetor $|\psi\rangle_{R}$ utilizando apenas três vetores dessa base, ou seja,

$$
|\psi\rangle_{R}=\cos (\xi)\left[\begin{array}{l}
1 \\
0
\end{array}\right]+\cos (\varphi) \operatorname{sen}(\xi)\left[\begin{array}{l}
0 \\
1
\end{array}\right]+\operatorname{sen}(\varphi) \operatorname{sen}(\xi)\left[\begin{array}{c}
0 \\
\mathrm{i}
\end{array}\right] .
$$

Isso conclui a demonstração.

O subespaço $\mathbb{V}$ de $\mathbb{C}^{2}(\mathbb{R})$, gerado pelos elementos

$$
\left\{\left[\begin{array}{l}
1 \\
0
\end{array}\right],\left[\begin{array}{l}
0 \\
1
\end{array}\right],\left[\begin{array}{l}
0 \\
\mathrm{i}
\end{array}\right]\right\}
$$


tem dimensão três. Como esse subespaço está definido sobre o corpo dos reais, ele é isomorfo a $\mathbb{R}^{3}$. Considere, então, um isomorfismo $\mathcal{T}$ entre $\mathbb{V}$ e $\mathbb{R}^{3}$, tal que

$$
\mathcal{T}\left(\left[\begin{array}{l}
0 \\
1
\end{array}\right]\right)=\left[\begin{array}{l}
1 \\
0 \\
0
\end{array}\right], \quad \mathcal{T}\left(\left[\begin{array}{l}
0 \\
\mathrm{i}
\end{array}\right]\right)=\left[\begin{array}{l}
0 \\
1 \\
0
\end{array}\right], \quad \mathcal{T}\left(\left[\begin{array}{l}
1 \\
0
\end{array}\right]\right)=\left[\begin{array}{l}
0 \\
0 \\
1
\end{array}\right]
$$

Usando esse isomorfismo, podemos escrever

$$
\mathcal{T}\left(|\psi\rangle_{R}\right)=\cos (\varphi) \operatorname{sen}(\xi)\left[\begin{array}{l}
1 \\
0 \\
0
\end{array}\right]+\operatorname{sen}(\varphi) \operatorname{sen}(\xi)\left[\begin{array}{l}
0 \\
1 \\
0
\end{array}\right]+\cos (\xi)\left[\begin{array}{l}
0 \\
0 \\
1
\end{array}\right]
$$

Podemos então imaginar que, quando desprezamos o fator de fase global de um q-bit, ele é "projetado" em um subconjunto de $\mathbb{R}^{3}$. Observamos que o lugar geométrico determinado por $|\psi\rangle_{R}$ (a partir do isomorfismo, podemos usar indistintamente $\mathcal{T}\left(|\psi\rangle_{R}\right)$ e $\left.|\psi\rangle_{R}\right)$ é uma semi-esfera de $\mathbb{R}^{3}$, denominada $S E^{2}$, com centro na origem e raio unitário (ou seja, $x^{2}+y^{2}+z^{2}=1$ e $z \geq 0$ ). Note que $0 \leq \xi \leq \pi / 2$ e $0 \leq \varphi<2 \pi$, como representamos na Figura 1(a).

Para chegarmos à esfera de Bloch, vamos enunciar dois lemas.

Lema 3.1. Sejam $\overline{S E}^{2}=S E^{2}-\left\{(0,0,1),(x, y, 0)^{4}\right\}$ (a semi-esfera na Figura 1(a) sem o equador e o pólo norte) e $Q=(0, \pi / 2) \times[0,2 \pi)$. A função a seguir é uma bijeção

$$
\begin{aligned}
f: Q & \rightarrow \overline{S E}^{2} \\
(\xi, \varphi) & \mapsto(\cos (\varphi) \operatorname{sen}(\xi), \operatorname{sen}(\varphi) \operatorname{sen}(\xi), \cos (\xi))
\end{aligned}
$$

Demonstração.

1) Sejam $X_{1}=\left(\xi_{1}, \varphi_{1}\right)$ e $X_{2}=\left(\xi_{2}, \varphi_{2}\right)$ em $Q \operatorname{com} f\left(X_{1}\right)=f\left(X_{2}\right)$.

Segue que:

$$
\left\{\begin{array}{l}
\cos \left(\varphi_{1}\right) \operatorname{sen}\left(\xi_{1}\right)=\cos \left(\varphi_{2}\right) \operatorname{sen}\left(\xi_{2}\right) \\
\operatorname{sen}\left(\varphi_{1}\right) \operatorname{sen}\left(\xi_{1}\right)=\operatorname{sen}\left(\varphi_{2}\right) \operatorname{sen}\left(\xi_{2}\right) \\
\cos \left(\xi_{1}\right)=\cos \left(\xi_{2}\right) .
\end{array}\right.
$$

Como $\xi_{1}, \xi_{2} \in(0, \pi / 2), \cos \left(\xi_{1}\right)=\cos \left(\xi_{2}\right) \Rightarrow \xi_{1}=\xi_{2} . \operatorname{Logo}, \operatorname{sen}\left(\xi_{1}\right)=\operatorname{sen}\left(\xi_{2}\right) \neq 0 ;$ substituindo no sistema acima, temos que $\cos \left(\varphi_{1}\right)=\cos \left(\varphi_{2}\right)$ e $\operatorname{sen}\left(\varphi_{1}\right)=\operatorname{sen}\left(\varphi_{2}\right)$. Como $\varphi_{1}, \varphi_{2} \in[0,2 \pi)$, $\log \varphi_{1}=\varphi_{2}$. E assim $X_{1}=X_{2}$ e $f$ é injetiva.

2) Como $\overline{S E}^{2}=S E^{2}-\{(0,0,1),(x, y, 0)\}$, com $x^{2}+y^{2}=1 \mathrm{e}$ $f(Q)=\left\{(x, y, z) \in \mathbb{R}^{3} ;(\cos (\varphi) \operatorname{sen}(\xi), \operatorname{sen}(\varphi) \operatorname{sen}(\xi), \cos (\xi)) \operatorname{com}(\xi, \varphi) \in Q\right\}$, queremos provar que $\overline{S E}^{2}=f(Q)$.

Vamos começar provando que $\overline{S E}^{2} \subset f(Q)$. Considerando $X=(x, y, z) \in \overline{S E}^{2}$, segue da definição dos conjuntos considerados que:

$$
x^{2}+y^{2}+z^{2}=1, \quad(x, y, z) \neq(0,0,1) \text { e } \quad z>0 .
$$

\footnotetext{
${ }^{4} \mathrm{Com} x^{2}+y^{2}=1$.
} 
Como $0<z<1$, então existe um $\xi \in(0, \pi / 2)$ tal que $\cos (\xi)=z$ e $\operatorname{sen}(\xi)=\sqrt{1-z^{2}}$. Como $x^{2}+y^{2}+z^{2}=1$ e $z \neq 1$, usando a identidade fundamental trigonométrica, podemos definir:

$$
\cos (\varphi)=\frac{x}{\sqrt{1-z^{2}}} \quad \text { e } \quad \operatorname{sen}(\varphi)=\frac{y}{\sqrt{1-z^{2}}} .
$$

Como $-\sqrt{1-z^{2}} \leq x \leq \sqrt{1-z^{2}}$ e $-\sqrt{1-z^{2}} \leq y \leq \sqrt{1-z^{2}}$, concluímos que $\varphi \in$ $[0,2 \pi)$. Logo, existe $(\xi, \varphi) \in Q$ para o qual $X=(\cos (\varphi) \operatorname{sen}(\xi), \operatorname{sen}(\varphi) \operatorname{sen}(\xi), \cos (\xi))$. Assim temos que $X \in f(Q)$ e que $\overline{S E}^{2} \subset f(Q)$.

Provando, agora, $f(Q) \subset \overline{S E}^{2}$.

Considerando $Y \in f(Q)$, então existe $(\xi, \varphi) \in Q$ tal que

$$
Y=(\cos (\varphi) \operatorname{sen}(\xi), \operatorname{sen}(\varphi) \operatorname{sen}(\xi), \cos (\xi)) .
$$

Como $\xi \in(0, \pi / 2)$ temos que $0<\cos (\xi)<1$. Assim, já que $(\xi, \varphi) \in Q$, deveremos ter $Y \neq(0,0,1)$. Substituindo as coordenadas de $Y$ na equação $x^{2}+y^{2}+z^{2}=1$, observamos que:

$$
\begin{aligned}
{[\cos (\varphi) \operatorname{sen}(\xi)]^{2}+[\operatorname{sen}(\varphi) \operatorname{sen}(\xi)]^{2}+[\cos (\xi)]^{2} } & = \\
=\left[\cos ^{2}(\varphi)+\operatorname{sen}^{2}(\varphi)\right] \operatorname{sen}^{2}(\xi)+\cos ^{2}(\xi) & =\operatorname{sen}^{2}(\xi)+\cos ^{2}(\xi)=1 .
\end{aligned}
$$

Então, $Y \in \overline{S E}^{2}$ e, segue daí que, $f(Q) \subset \overline{S E}^{2}$. Logo, $f(Q)=\overline{S E}^{2}$ e $f$ é sobrejetiva.

Concluímos, então, que $f$ é uma bijeção.

De forma análoga, podemos provar que

Lema 3.2. Sejam $\bar{S}^{2}=S^{2}-\{(0,0,1),(0,0,-1)\}$ (a esfera na Figura 1(b) sem os pólos norte e sul) e $Q=(0, \pi / 2) \times[0,2 \pi)$. A função

$$
\begin{aligned}
g: Q & \rightarrow \bar{S}^{2} \\
(\xi, \varphi) & \mapsto(\cos (\varphi) \operatorname{sen}(2 \xi), \operatorname{sen}(\varphi) \operatorname{sen}(2 \xi), \cos (2 \xi))
\end{aligned}
$$

é uma bijeção.

Proposição 3.2. Sejam $f$ e $g$ as funções definidas nos Lemas 3.1 e 3.2, respectivamente. A função abaixo é sobrejetora e, para $z \neq 0$, também é injetora.

$$
\begin{aligned}
\mathcal{V}: S E^{2} & \rightarrow S^{2} \\
(x, y, z) & \mapsto g\left(f^{-1}(x, y, z)\right) \quad\left((x, y, z) \in \overline{S E}^{2}\right) \\
(0,0,1) & \mapsto(0,0,1) e \quad(x, y, 0) \mapsto(0,0,-1)
\end{aligned}
$$

Demonstração. Usando os Lemas 3.1 e 3.2 e observando que o domínio da função $g$ é igual ao contra-domínio da função $f^{-1}$, obtemos uma bijeção entre $\overline{S E}^{2}$ e $\bar{S}^{2}$, dada por $g \circ f^{-1}$, já que a composição de bijeções, nessas condições, é uma bijeção. A função $\mathcal{V}$, definida entre $S E^{2}$ e $S^{2}$, é, então, uma função sobrejetora e, para $z \neq 0$, também é injetora, pela sua própria construção. 


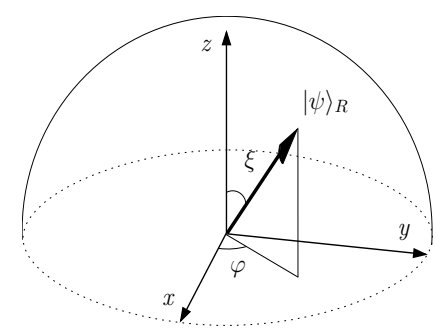

(a) Semi-esfera que é o local geométrico de todos os q-bits $|\psi\rangle_{R}$.

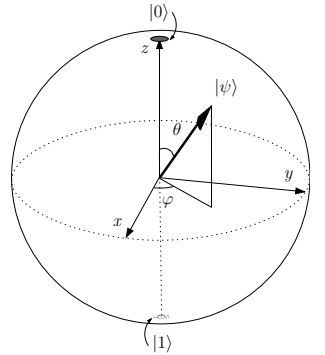

(b) Esfera de Bloch com representação de um q-bit genérico $|\psi\rangle$ e dos q-bits $|0\rangle$ e $|1\rangle$.

Figura 1: Semi-esfera e esfera de Bloch.

Corolário 3.1. A função $\mathcal{V}$ tem uma formulação analítica explícita dada por

$$
\begin{array}{ll}
\mathcal{V}: S E^{2} & \rightarrow S^{2} \\
(x, y, z) & \mapsto\left(2 x z, 2 y z, 2 z^{2}-1\right) .
\end{array}
$$

Cada elemento da imagem de $\mathcal{V}$ será chamado de vetor de Bloch.

Definição 3.1 (Esfera de Bloch). A esfera de Bloch é o lugar geométrico de todos os vetores de Bloch.

\section{Observações:}

1. Os elementos da base computacional $\{|0\rangle,|1\rangle\}$ são representados na esfera de Bloch pelos pólos norte $(0,0,1)$ e sul $(0,0,-1)$, respectivamente.

2. $\mathcal{V}$ leva todos os pontos do equador de $S E^{2}$ no pólo sul $(0,0,-1)$ de $S^{2}$.

3. Fazendo a mudança de variável $\xi \rightarrow \frac{\theta}{2}$, na equação (2.1), chegamos à forma para a representação polar de um q-bit mais comum na literatura da área:

$$
|\psi\rangle=\mathrm{e}^{i \gamma}\left[\cos \left(\frac{\theta}{2}\right)|0\rangle+\mathrm{e}^{i \varphi} \operatorname{sen}\left(\frac{\theta}{2}\right)|1\rangle\right] .
$$

E, para o vetor de Bloch, teremos:

$$
|\psi\rangle_{B}=(\cos \varphi \operatorname{sen} \theta, \operatorname{sen} \varphi \operatorname{sen} \theta, \cos \theta), \quad 0 \leq \varphi<2 \pi, \quad 0 \leq \theta \leq \pi .
$$

4. Cada ponto da esfera de Bloch representa todos os q-bits de formato $\mathrm{e}^{i \gamma}|\psi\rangle$, para $|\psi\rangle=\left[\cos (\theta / 2)|0\rangle+\mathrm{e}^{i \varphi} \operatorname{sen}(\theta / 2)|1\rangle\right]$ fixado e $0 \leq \gamma<2 \pi$.

Na Figura 1(b), apresentamos uma representação da esfera de Bloch. 


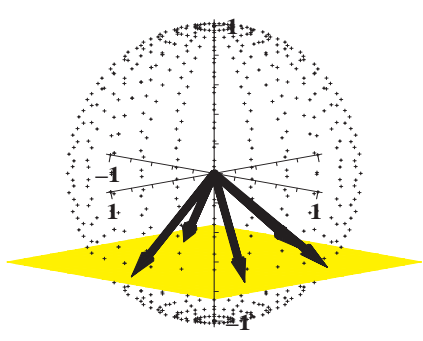

(a) Vários vetores de Bloch com mesmo $\theta$.

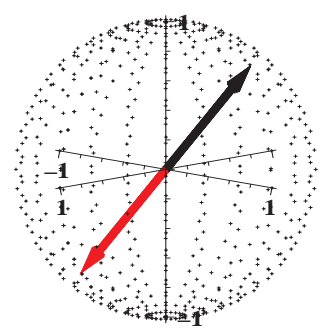

(b) Dois vetores de Bloch antípodas e ortogonais.

Figura 2: Representação gráfica das Propriedades 4.1 e 4.2 .

\section{Algumas Propriedades da Esfera de Bloch}

O principal objetivo da representação de um q-bit na esfera de Bloch é possibilitar alguma visualização da evolução desse q-bit no tempo. Pela sua própria construção, a esfera de Bloch apresenta várias propriedades que não são intuitivas. Dentre essas, destacaremos duas.

Propriedade 4.1 (q-bits equiprováveis). Na esfera de Bloch, usando a parametrização apresentada em (3.1), quaisquer dois vetores de Bloch que pertençam a um mesmo plano, paralelo ao plano $X Y$, representam q-bits que têm probabilidades iguais de produzirem $|0\rangle$ ou $|1\rangle$, ao serem medidos.

Demonstração. Os representantes dos q-bits

$$
|\psi\rangle_{1}=\alpha_{1}|0\rangle+\beta_{1}|1\rangle \quad \text { e } \quad|\psi\rangle_{2}=\alpha_{2}|0\rangle+\beta_{2}|1\rangle
$$

que estão em um mesmo plano paralelo a $X Y$, têm, respectivamente, $\theta_{1}$ associado a $|\psi\rangle_{1}$ e $\theta_{2}$ associado a $|\psi\rangle_{2}$, relativos à equação (3.1). Então, $\theta_{1}=\theta_{2}$ e

$$
\left|\alpha_{1}\right|=\cos \left(\frac{\theta_{1}}{2}\right)=\cos \left(\frac{\theta_{2}}{2}\right)=\left|\alpha_{2}\right| \quad \text { e } \quad\left|\beta_{1}\right|=\operatorname{sen}\left(\frac{\theta_{1}}{2}\right)=\operatorname{sen}\left(\frac{\theta_{2}}{2}\right)=\left|\beta_{2}\right| .
$$

Ver representação na Figura 2(a).

Propriedade 4.2 (vetores de Bloch antípodas e ortogonais). Na esfera de Bloch, usando a parametrização apresentada em (3.1), quaisquer dois vetores de Bloch antípodas são representantes de dois q-bits ortogonais.

Demonstração. As famílias de q-bits relativas a vetores de Bloch antípodas são definidas por

$$
\begin{aligned}
& |\phi\rangle_{1}=\mathrm{e}^{\left(\mathrm{i} \gamma_{1}\right)}\left(\cos \left(\frac{\theta_{1}}{2}\right)|0\rangle+\mathrm{e}^{\left(\mathrm{i} \varphi_{1}\right)} \operatorname{sen}\left(\frac{\theta_{1}}{2}\right)|1\rangle\right) \mathrm{e} \\
& |\phi\rangle_{2}=\mathrm{e}^{\left(\mathrm{i} \gamma_{2}\right)}\left(\cos \left(\frac{\pi-\theta_{1}}{2}\right)|0\rangle+\mathrm{e}^{\mathrm{i}\left(\varphi_{1}+\pi\right)} \operatorname{sen}\left(\frac{\pi-\theta_{1}}{2}\right)|1\rangle\right) .
\end{aligned}
$$


Observe que esses q-bits, quando representados na esfera de Bloch, são antípodas no sentido usual do termo.

Reescrevendo $|\phi\rangle_{2}$, temos:

$$
|\phi\rangle_{2}=\mathrm{e}^{\left(\mathrm{i} \gamma_{2}\right)}\left(\operatorname{sen}\left(\frac{\theta_{1}}{2}\right)|0\rangle+\mathrm{e}^{\mathrm{i}\left(\varphi_{1}+\pi\right)} \cos \left(\frac{\theta_{1}}{2}\right)|1\rangle\right) .
$$

Calculando o produto interno usual em $\mathbb{C}^{2}(\mathbb{C})$ entre $|\phi\rangle_{1}$ e $|\phi\rangle_{2}$, obtemos:

$$
\begin{aligned}
\mathrm{e}^{\mathrm{i}\left(\gamma_{1}-\gamma_{2}\right)}\left(\cos \left(\frac{\theta_{1}}{2}\right) \operatorname{sen}\left(\frac{\theta_{1}}{2}\right)+\mathrm{e}^{\mathrm{i}\left(\varphi_{1}-\varphi_{1}-\pi\right)} \cos \left(\frac{\theta_{1}}{2}\right) \operatorname{sen}\left(\frac{\theta_{1}}{2}\right)\right) & = \\
& =\mathrm{e}^{\mathrm{i}\left(\gamma_{1}-\gamma_{2}\right)}\left(\cos \left(\frac{\theta_{1}}{2}\right) \operatorname{sen}\left(\frac{\theta_{1}}{2}\right)\left(1+\mathrm{e}^{-\mathrm{i} \pi}\right)\right)=0 .
\end{aligned}
$$

Ou seja, $|\phi\rangle_{1}$ e $|\phi\rangle_{2}$ são ortogonais (ver representação na Figura 2(b)).

Com isso, apresentamos uma descrição matemática e algumas propriedades da esfera de Bloch. Em outros trabalhos, estudaremos a ação de algumas portas quânticas em q-bits usando a visualização permitida pela esfera de Bloch. Em paralelo, estamos estudando outras representações para 1 e 2 q-bits.

\section{Agradecimentos}

Os autores agradecem o apoio da FAPERJ, da FAPESP, do CNPq, e da aluna Virgínia Silva da Costa, pela rotina que produziu as representações gráficas dos q-bits na esfera de Bloch. Agradecem também a um revisor pois seus comentários contribuíram para aclarar alguns pontos nebulosos do texto.

Abstract. We present a mathematical description of the Bloch sphere and discuss some properties of this representation. Also, using the Bloch sphere, we represent some qubits with special properties.

\section{Referências}

[1] W.E. Baylis, R. Cabrera, C. Rangan, Control and representation of n-qubit quantum systems, arxiv.org, quant-ph/0606019, June 2006.

[2] B.A. Bernevig, H.-D. Chen, Geometry of the three-qubit state, entanglement and division algebras, J. Ph. A: Math. and Gen.l, 36 (2003), 8325-8339.

[3] K. Dietz, Generalized Bloch spheres for m-qubit states, Journal of Physics A: Mathematical and General, 3 (2006), 1433-1447.

[4] R.P. Feynman, F.L. Vernon, R.W. Hellwarth, Geometrical representation of the Schröedinger equation for solving the MASER problem, Journal of Applied Physics, 28, No. 1 (1957), 49-52.

[5] T. Havel, C. Doran, A Bloch-sphere-type model for two qubits in the geometric algebra of a 6-D Euclidean vector space, Proceedings of SPIE, 5436 (2004), 93-106. 
[6] M. Hirvensalo, "Quantum Computing", Springer, New York, 2001.

[7] K. Kato, M. Oto, H. Imai, K. Imai, Voronoi diagrams for pure 1-qubit quantum states, arxiv.org, Quantum Physics, quant-ph/0604101, April 2006.

[8] G. Kimura, A. Kossakowski, The Bloch-vector space for n-level systems: the spherical-coordinate point of view, Open Systems 8 Information Dynamics, 12, No. 3 (2005), 207-229.

[9] A. Y. Kitaev, A. Shen, M. Vyalyi, "Classical and Quantum Computing", volume 47 of Graduate Studies in Mathematics, AMS, 2002.

[10] P. Kurzynski, A. Grudka, Graphical representation of generalized quantum measurements, arxiv.org, Quantum Physics, quant-ph/0604189, April 2006.

[11] A. Maitra, P. Parashar, Hadamard type operations for qubits, arxiv.org, Quantum Physics, quant-ph/0505068, May 2005.

[12] R. Mosseri, Two and three qubits geometry and Hopf fibrations, arxiv.org, Quantum Physics, quant-ph/0310053, 2003.

[13] M. A. Nielsen, I. L. Chuang, "Quantum Computation and Quantum Information", Cambridge University Press, Cambridge, 2000.

[14] R. Portugal, C. Lavor, L. M. Carvalho, N. Maculan, "Uma Introdução à Computação Quântica", Notas em Matemática Aplicada, Vol. 8, SBMAC, São Carlos, 2004.

[15] J. Preskill, "Quantum Information and Computation", Lecture Notes, California Institute of Technology, unpublished 1998.

[16] J. Zhang, J. Vala, S. Sastry, K.B. Whaley, Geometric theory of nonlocal twoqubit operations, Phys. Rev. A, 67, No. 4 (2003), 042313. 